\title{
Nanoscale Electrochemistry
}

\author{
R. Schuster,* V. Kirchner, X.H. Xia, A. M. Bittner, ${ }^{\dagger}$ and G. Ertl \\ Fritz-Haber-Institut der Max-Planck-Gesellschaft, Faradayweg 4-6, D-14195 Berlin, Germany
}

(Received 16 December 1997)

\begin{abstract}
The lateral extension of electrochemically induced surface modifications is usually determined by the macroscopic size of the electrodes and the diffusion length of the reacting species. To overcome this constraint, we conducted an electrochemical reaction far from equilibrium. We applied short voltage pulses ( $\leq 100 \mathrm{~ns}$, up to $\pm 4 \mathrm{~V}$ ) to a scanning tunneling microscope tip while imaging a Au(111) surface in concentrated electrolytes. They lead either to hole formation by anodic dissolution of the Au or to cathodic deposition of $\mathrm{Cu}$ islands (in the $\mathrm{Cu}^{2+}$ containing electrolyte), both of nanometer extension. [S0031-9007(98)06426-6]
\end{abstract}

PACS numbers: 68.35.Bs, 82.40.Js, 82.45.+z

Soon after the development of scanning probe microscopic techniques, various attempts were undertaken to use the tip as a tool for modifying the morphology of surfaces on a nanometer length scale [1-3]. These experiments comprised the manipulation of adsorbed particles or the removal of substrate atoms [4-7] as well as the controlled deposition of tip material (see, e.g., [8]). Nanostructuring was also achieved by involving electrochemical processes into the overall procedures. For example, Li et al. $[9,10]$ deposited Ag and Pt clusters on a graphite surface by applying positive voltage pulses to the STM tip in a solution containing the respective metal ions. This effect was attributed to nucleation within holes which were created on the substrate by the voltage pulses, presumably by field desorption. Kolb et al. [11], on the other hand, were able to detach $\mathrm{Cu}$ clusters from a tip, where they had been previously deposited electrochemically, onto a Au substrate by mechanical contact.

All of these techniques suffer from restrictions (e.g., concerning the deposited material or destruction of the substrate) which could be largely avoided if controlled nanostructuring could be achieved by a direct local electrochemical reaction on the substrate, with the geometry being determined by the location of the tip which acts as a local counter electrode. A similar approach has been employed in the scanning electrochemical microscope where a microelectrode was used to electrochemically modify surfaces on a micrometer scale [12]. These ideas are, however, hampered by a serious problem: The electrochemical reaction is governed by the potential drop in the double layer and the chemical composition within a very narrow region at the electrode (the so-called inner Helmholtz layer) [13]. Application of an external voltage causes rapid adjustment of the distribution of ions within this double layer, and, as a consequence, the potential varies essentially across the Helmholtz layers of the working and counter electrode. Thus, the geometry of the tip-substrate configuration becomes unimportant, and the electrochemical reaction is expected not to be confined to the apex region of the tip, as also actually observed. The present paper describes a possibility to overcome this intrinsic problem: The system is prevented from reaching the outlined equilibrium situation by applying voltage pulses of only a very short duration $(\leq 100 \mathrm{~ns})$. In this way, holes of about $5 \mathrm{~nm}$ diameter and 0.3 to $1 \mathrm{~nm}$ depth on a Au substrate were created by local anodic dissolution, while cathodic polarization led to the deposition of small $\mathrm{Cu}$ clusters.

As a sample, a $250 \mathrm{~nm}$ thick Au film, evaporated onto a glass substrate, was used. It was flame annealed prior to the experiment. High resolution STM images exhibited flat terraces with up to $100 \mathrm{~nm}$ width on which the $\sqrt{3} \times 22$ reconstruction of the clean $\mathrm{Au}(111)$ surface [14] was discernible. The experiments were conducted in situ in an electrochemical STM, where the potential of the sample against the reference electrode $\left(\mathrm{Cu} / \mathrm{Cu}^{2+}\right)$ was controlled by a potentiostat while a Pt wire served as the counter electrode [15]. Indicated electrochemical potentials refer to the standard hydrogen electrode (SHE). In the tunneling mode, a voltage $U_{T}$ was applied between the tip and the surface. STM tips were made from electrochemically inert Ir wires by mechanical grinding and were covered with Apiezon wax W100, except for the very apex of the tip, to reduce Faradaic currents. For conducting local electrochemistry, a pulse generator which provided pulses with variable duration down to $10 \mathrm{~ns}$ and with voltages up to $\pm 4 \mathrm{~V}$ was switched onto the tip. In this way, only the immediate vicinity of the tip region was subjected to potential changes. During the application of the pulses, the feedback loop of the STM was switched off, except for Fig. 2 (below).

Figure 1(a) shows an STM image after applying a voltage of $-4 \mathrm{~V}$ to the tip for $10 \mu \mathrm{s}$. The Au surface, which initially consisted of atomically flat terraces separated by monoatomic steps, now exhibits a pattern characteristic for the large-scale electrochemical oxidation of $\mathrm{Au}$, essentially consisting of small holes and hills with monoatomic vertical extension [16-18]. No local features which could be attributed to the location of the tip are discernible, in accordance with the general remarks on the potential distribution made above. However, reduction of the pulse length continuously decreases the diameter of the oxidized region. Upon applying pulses of 600 and 200 ns duration, the 

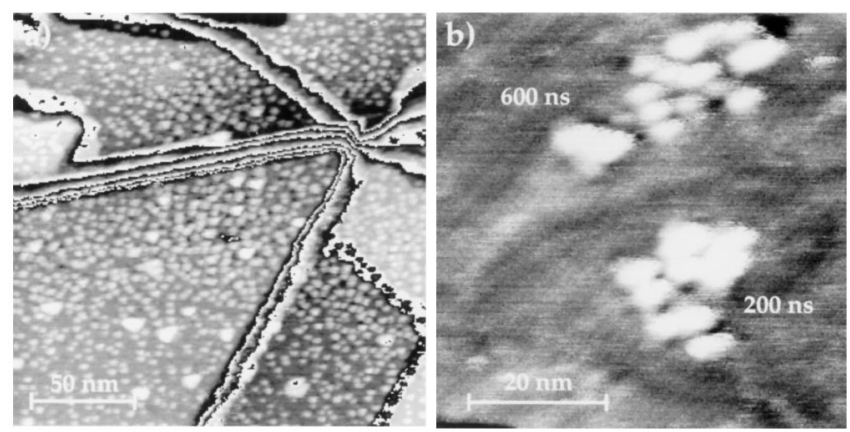

FIG. 1. (a) Large-scale oxidation of the $\mathrm{Au}(111)$ surface upon a voltage pulse of $10 \mu \mathrm{s}$ duration and $-4 \mathrm{~V}$ voltage. Terraces were colored individually. (b) Shorter pulse durations (indicated in the figure) lead to smaller oxidized regions $(0.5 \mathrm{M}$ $\mathrm{CuSO}_{4} ; U_{S}=740 \mathrm{mV} ; U_{T}=-200 \mathrm{mV} ; I_{T}=1 \mathrm{nA}$.

oxidized region shrank to about 30 and $15 \mathrm{~nm}$ in diameter [Fig. 1(b)]. It should be noted here that the tip has to withstand the high voltage pulses without substantial mechanical changes. This requirement sometimes degrades the resolution of the tip for recording the STM images. Figure 1(b) also rules out the possibility that the features are simply caused by mechanical contact between the tip and the surface. Mechanical contact would cause a single hole at the surface and not the hole and island patches as discernible in Fig. 1(b) which are typical for electrochemical oxidation. Hence, the electrochemical reaction becomes indeed localized by reducing the pulse duration into the nanosecond range.

Even shorter pulses lead to the creation of localized holes confined to the region under the tip, as demonstrated by Fig. 2. In Fig. 2(a), arrows mark the position of the tip during the application of three pulses with $-2 \mathrm{~V}$ voltage and $60 \mathrm{~ns}$ duration. The slow scan direction runs upwards, and, hence, only the upper part of the holes is imaged since these are formed directly below the tip. (The bright features in the right part were already present prior to application of the voltage pulses.) The subsequent image [Fig. 2(b)] exhibits the complete holes whose diameters vary slightly around $5 \mathrm{~nm}$ while their depth amounts to 1 to 3 monolayers (ML). The holes exhibit flat bottoms, as shown for the uppermost hole by the cross section in Fig. 2(c). Large-scale images demonstrated that no additional structures had formed on the surface; i.e., the nanostructuring is indeed confined to the narrow region where the voltage pulse to the tip is applied. The duration of the pulses is about $10^{5}$ times smaller than the time needed for scanning a horizontal line of the STM image. Therefore, the tip practically did not move during the duration of the pulses, i.e., the hole formation process, and the reaction is well localized around the very apex of the tip where the tunneling occurs.

Figure 2(d) shows the electronic response of the STM control upon creating the uppermost hole in Fig. 2(a). The time for recording one line of the STM image from left to right was $90 \mathrm{~ms}$. As the input of the current amplifier re-
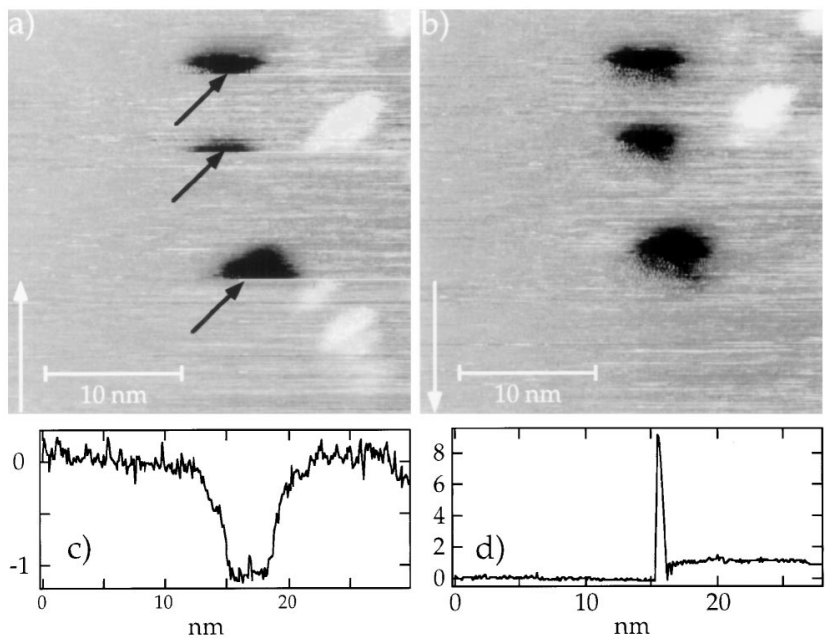

FIG. 2. Formation of holes by $-2 \mathrm{~V}$ pulses of $60 \mathrm{~ns}$ duration. (a) In situ formation of three holes by three pulses. The black arrows indicate the tip position during the pulses. The scan direction is also indicated (white arrows). (b) Subsequent STM image showing the complete holes $\left(U_{S}=840 \mathrm{mV} ; U_{T}=\right.$ $-300 \mathrm{mV} ; I_{T}=1 \mathrm{nA} ; 1 \mathrm{M} \mathrm{CuSO}_{4} / 0.5 \mathrm{M} \mathrm{H}_{2} \mathrm{SO}_{4}$ ). (c) Cross section through the uppermost hole in (b). (d) Response of the tip during the creation of the uppermost hole in (a) ( $z$ axes in ML).

mained connected to the tip during the application of the voltage pulse, the amplifier was overloaded, and the feedback loop started to retract the tip $\approx 1 \mathrm{~ms}$ after the application of the $60 \mathrm{~ns}$ pulse. After $\approx 5 \mathrm{~ms}$ of recovery time, the tip approached the surface again, continuing its scan line approximately $1 \mathrm{ML}$ higher than before. This indicates a slight elongation of the tip. However, as the distance between the tip and the surface amounts to about $4 \mathrm{ML}$ (as shown later), no mechanical contact occurred during the hole formation. By gating the feedback loop, the response of the STM control can be significantly suppressed, which, however, has no influence on the hole formation.

Systematic experiments with a variation of the electrochemical parameters revealed that hole formation generally requires a negative threshold voltage of the order of -1.5 to $-2.5 \mathrm{~V}$. Positive pulses of up to $5 \mathrm{~V}$ did not result in the formation of holes. The formation of holes by short $(\leq 100 \mathrm{~ns})$ negative voltage pulses works best in highly concentrated electrolytes (e.g., $\geq 1 \mathrm{M} \mathrm{KCl}$ or $\mathrm{CuSO}_{4}$ ). In pure $\mathrm{H}_{2} \mathrm{O}$ or in $10 \mathrm{mM} \mathrm{CuSO}_{4}$ solution, no holes were created with $100 \mathrm{~ns}$ pulses. This is in accordance with previous reports in the literature $[19,20]$, according to which tip induced holes can be created on Au surfaces in humid air only with pulses longer than $1 \mathrm{~ms}$. A possible mechanism underlying these latter observations will be discussed later.

Figure 3(a) shows an example for the opposite type of reaction, namely, the cathodic reduction of metal ions, leading to the deposition of small clusters of nanometer scale. In an electrolyte consisting of $1 \mathrm{M} \mathrm{CuSO}_{4}$ and $0.5 \mathrm{M} \mathrm{H}_{2} \mathrm{SO}_{4}$, the surface was held at a potential of $U_{S}=$ $410 \mathrm{mV}$ vs SHE where bulk $\mathrm{Cu}$ cannot be deposited: The Nernst potential for $\mathrm{Cu}$ bulk deposition is $340 \mathrm{mV}$. 

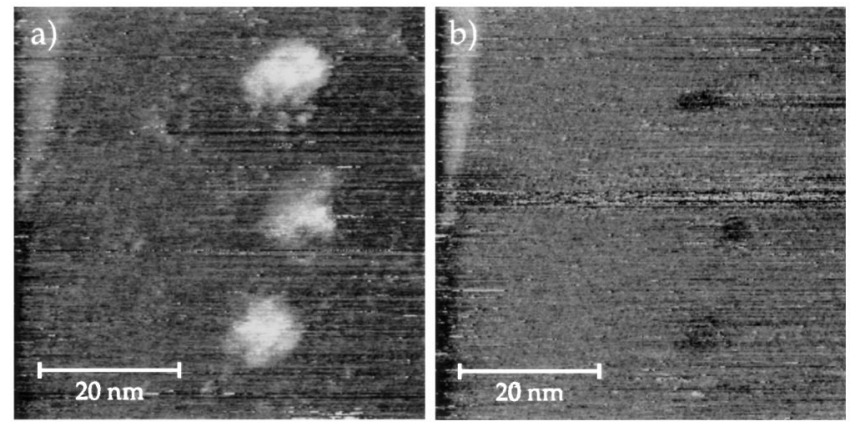

FIG. 3. (a) Formation of $\mathrm{Cu}$ islands by $3 \mathrm{~V}$ pulses with $50 \mathrm{~ns}$ duration $\left(U_{S}=410 \mathrm{mV} ; U_{T}=230 \mathrm{mV}\right)$. (b) After dissolution of the $\mathrm{Cu}$ clusters at $U_{S}=740 \mathrm{mV}\left(U_{T}=-100 \mathrm{mV}\right)$. The shallow indentations appear about $\frac{1}{5} \mathrm{ML}$ deep $\left(I_{T}=1 \mathrm{nA}\right.$; $1 \mathrm{M} \mathrm{CuSO}_{4} / 0.5 \mathrm{M} \mathrm{H}_{2} \mathrm{SO}_{4}$ ).

Application of positive voltage pulses of $3 \mathrm{~V}$ with $50 \mathrm{~ns}$ duration to the tip leads to the deposition of $\mathrm{Cu}$ clusters with about $8 \mathrm{~nm}$ diameter and 1 to $2 \mathrm{ML}$ height, located at the actual position where the pulse was applied. As the sample potential is about $70 \mathrm{mV}$ more positive than that for the bulk $\mathrm{Cu}$ deposition, the $\mathrm{Cu}$ clusters are unstable and slowly dissolve again on a time scale of several minutes. Figure 3(b) shows the surface after complete dissolution and restoration of the clean surface at $U_{S}=740 \mathrm{mV}$, by which the chemical nature of the deposit is identified as metallic $\mathrm{Cu}$. In Fig. 3(b), the former positions of the clusters are still visible as shallow indentations, appearing about $\frac{1}{5}$ ML deep. However, these remainders showed up only in some experiments and depend on experimental conditions, i.e., the observation time of the clusters. In agreement with Ullmann et al., we interpret this as an indication for the beginning alloy formation between $\mathrm{Au}$ and $\mathrm{Cu}$, leading to distortions of the surface top layer [21]. In none of these cases were holes found comparable to those in Fig. 2. We therefore attribute the formation of the clusters to a local electrochemical reaction rather than to the nucleation of clusters in surface defects, as, e.g., demonstrated by $\mathrm{Li}$ et al. for $\mathrm{Ag}$ on graphite $[9,10]$. Note that there the deposition of the metal deposits started only several microseconds after the beginning of the pulse, and the applied voltages amounted up to $6 \mathrm{~V}$. Additionally, in these experiments, the electrolyte contained only $0.5 \mathrm{mM}$ $\mathrm{Ag}^{+}$ions. Therefore, the deposited $\mathrm{Ag}$ atoms are supposed to stem from an underpotentially deposited (UPD) Ag layer on the STM tip, rather than from the bulk of the solution [10]. In our experiment, however, besides a very high ion concentration in the electrolyte, the tip was held at $640 \mathrm{mV}$ vs SHE, while recording Fig. 3. At this potential, UPD $\mathrm{Cu}$ is not expected to form on the tip. Hence, for the formation of the dense clusters, $\mathrm{Cu}$ ions from the solution had to be reduced during the voltage pulses. Again, the short duration of the voltage pulses is absolutely necessary for the formation of localized structures. This is shown in Fig. 4 where a pulse of $1.9 \mathrm{~V}$ was applied to the tip for $20 \mu$ s which resulted in the nucleation of a large number of $\mathrm{Cu}$ clusters scattered over the whole imaged surface area.

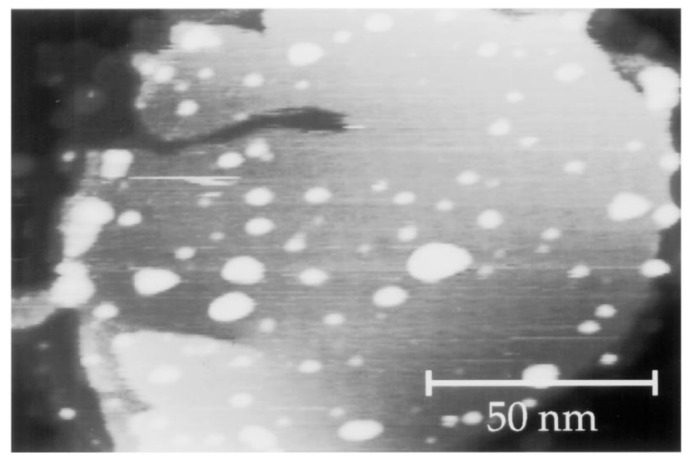

FIG. 4. Large-scale $\mathrm{Cu}$ deposition after a $20 \mu \mathrm{s} \times 1.9 \mathrm{~V}$ pulse in $0.5 \mathrm{M} \mathrm{CuSO}_{4}\left(U_{T}=50 \mathrm{mV} ; I_{T}=1 \mathrm{nA}\right)$.

After demonstrating that, by applying very short ( $\leq 100 \mathrm{~ns}$ ) voltage pulses to an STM tip, localized structures can indeed be created, the need for a plausible explanation of this effect becomes obvious. In gas phase or vacuum experiments, the most likely mechanism underlying the local removal of substrate atoms is chemically assisted field desorption $[5,6,22,23]$. There the STM tip is so close to the surface (even closer than atomic distances) that chemical interactions between the tip and the surface come into play, and the field strength necessary for the release of atoms is considerably reduced if compared to "normal" field evaporation [6].

In order to check such a possibility for the hole formation in the present study, the tip-surface separation was determined by recording the tunneling current as a function of distance. For typical conditions applied for hole formation $\left(I_{T}=1 \mathrm{nA}, U_{T}=-200 \mathrm{mV}\right)$ the working distance was determined to be rather large, namely, about $1 \mathrm{~nm}$. The resulting low threshold field for the hole formation of only $2 \mathrm{~V} / \mathrm{nm}$ rules out the above-mentioned chemically assisted field desorption mechanism. We rather have to concentrate on the possibility of an electrochemical mechanism. Indeed, the hole and island patches in Fig. 1(b) are a fingerprint for electrochemical Au oxidation.

The rather blunt Ir tip has a radius of about $500 \mathrm{~nm}$ so that the electrolyte is confined to a narrow gap of only a few nanometers width extending over about $100 \mathrm{~nm}$, where tunneling is enabled by only a few atoms protruding from the flat body of the tip. Assuming a typical capacity of the double layer of $10 \mu \mathrm{F} / \mathrm{cm}^{2}$, its polarization by $1 \mathrm{~V}$ requires the accumulation of $\approx 10^{14}$ ions $/ \mathrm{cm}^{2}$, which is equivalent to about 0.1 ML. Since the gap is only a few nanometers wide, the buildup of the double layer will almost instantaneously largely deplete the electrolyte from ions. (A typical electrochemical mobility of $10^{-4} \mathrm{~cm}^{2} / \mathrm{V} \mathrm{s}$ [13] leads to a subnanosecond time scale for migration of ions across the gap. Other transport mechanisms such as diffusion or convection do not play important roles at such length scales as the gap width.) As a consequence of the depletion, the electrolyte resistance rises, and electrochemical current flow (which would follow the reduction/ oxidation of species in the double layer) will be suppressed. Only with progressing time will this region be refilled by 
ions diffusing into the gap from the bulk solution adjacent to the tip region. This is the reason why for longer pulses large-scale reaction is observed. With a typical value of $10^{-5} \mathrm{~cm}^{2} / \mathrm{s}$ for the diffusion constant of ions in aqueous solution [13], the diffusion length is estimated to be about $10 \mathrm{~nm}$ within $100 \mathrm{~ns}$, which is indeed smaller than the extension of the gap region. Therefore, only for very short pulses, the ionic depletion is maintained during the duration of the pulse, and the electrochemical reaction is largely suppressed. Why, however, is this process taking place in the immediate vicinity of the location where the tunneling producing the STM images takes place? In this region, the two electrodes are only $1 \mathrm{~nm}$ or a few water molecules apart, and, hence, the concept of two double layers separated by an electrolyte breaks down. Rather, the two double layers merge, and the notion of an electrolyte resistance becomes meaningless. Ions created electrochemically at the apex of the tip are instantaneously transported by the local electric field to the sample surface and vice versa. This effect will be confined to the region where the two electrodes are closest to each other (where also the STM process takes place) and accounts for the strongly localized character of the structures formed. Thus, for example, upon oxidation of the surface, Au ions are transported directly to the tip. There they are reduced and form an additional metallic layer on the tip. This obviously happened in Fig. 1(d), where the tip elongates by approximately $1 \mathrm{ML}$.

The proposed mechanism seems to be in contradiction to the quoted observation of hole formation on Au surfaces in humid air $[19,20]$. There the reaction was reported to start at about $18 \%$ humidity, from which the existence of an about $2 \mathrm{~nm}$ thick water layer was concluded, and an electrochemical mechanism was proposed. However, for reasonably low threshold voltages, long voltage pulses $(>100 \mathrm{~ms})$ were required in order to create holes. This finds its natural explanation in the very low ion concentration $\left(10^{-7} \mathrm{M}\right.$ in pure water) which would prevent any double layer formation during short pulses. In this case, the lateral confinement of the reaction has rather to be attributed to the finite amount of electrolyte forming a "neck" between the tip and the sample by capillary forces. This is in agreement with the conclusions drawn in experiments on oxidation of silicon [24] and Ti [25]. Indeed, in experiments with bulk water, we found large-scale oxidation already upon application of microsecond long voltage pulses.

The present results lead to the conclusion that for "true" nanoscale electrochemistry the ion concentration should be high in order to enable the buildup of the double layers in the small gap between the tip and the sample during the short voltage pulses. The short duration of the pulses prevents lateral diffusion of ions into the small tip-surface gap, which would lead to large-scale modifications as in ordinary electrochemistry. The principle underlying the local confinement of electrochemical reactions appears to be quite universal and is considered to open a route for wide applications.
The authors gratefully acknowledge fruitful discussions with P. Allongue, K. Doblhofer, K. Krischer, M. Orlik, and K. G. Weil. We thank A. Martin and K. Grabitz for the preparation of the samples.

*Author to whom correspondence should be addressed. Electronic address: Schuster_R@FHI-Berlin.mpg.de

${ }^{\dagger}$ Present address: Institut de Physique Expérimentale (IPE), DP-EPFL, CH-1015 Lausanne, Switzerland.

[1] U. Staufer, in Scanning Tunneling Microscopy II, edited by H.-J. Güntherodt and R. Wiesendanger (SpringerVerlag, Berlin, 1992), p. 273.

[2] R. Wiesendanger, J. Vac. Sci. Technol. B 12, 515 (1994).

[3] R.M. Nyffenegger and R.M. Penner, Chem. Rev. 97, 1195 (1997).

[4] D. M. Eigler and E. K. Schweizer, Nature (London) 344, 524 (1990).

[5] J. A. Stroscio and D. M. Eigler, Science 254, 1319 (1991).

[6] P. Avouris and I. Lyo, Appl. Surf. Sci. 60/61, 426 (1992).

[7] G. Meyer, L. Bartels, S. Zöphel, E. Henze, and K. Rieder, Phys. Rev. Lett. 78, 1512 (1997).

[8] H. J. Mamin, S. Chiang, H. Birk, P. H. Guethner, and D. Rugar, J. Vac. Sci. Technol. B 9, 1398 (1991).

[9] W. Li, J. A. Virtanen, and R. M. Penner, Appl. Phys. Lett. 60, 1181 (1992).

[10] W. Li, G. S. Hsiao, D. Harris, R. M. Nyffenegger, J. A. Virtanen, and R. M. Penner, J. Phys. Chem. 100, 20103 (1996).

[11] D. M. Kolb, R. Ullmann, and T. Will, Science 275, 1097 (1997).

[12] D. M. Mandler and A. J. Bard, J. Electrochem. Soc. 137, 1079 (1990).

[13] J. O’M. Bockris and A. K. N. Reddy, Modern Electrochemistry (Plenum, New York, 1970).

[14] W. Haiss, D. Lackey, J. K. Sass, and K. H. Besocke, J. Chem. Phys. 95, 2193 (1991).

[15] A. M. Bittner, J. Wintterlin, and G. Ertl, J. Electroanal. Chem. 388, 225 (1995).

[16] H. Hondo, S. Sugawara, and K. Itaya, Anal. Chem. 62, 2424 (1990).

[17] X. Gao and M. J. Weaver, J. Electroanal. Chem. 367, 259 (1994).

[18] C. M. Vitus and A. J. Davenport, J. Electrochem. Soc. 141, 1291 (1994).

[19] C. Lebreton and Z.Z. Wang, Scanning Microsc. 8, 441 (1994).

[20] C. Lebreton and Z.Z. Wang, J. Vac. Sci. Technol. B 14, 1356 (1996).

[21] R. Ullmann, T. Will, and D. M. Kolb, Chem. Phys. Lett. 209, 238 (1993).

[22] G. Meyer, S. Zöphel, and K.H. Rieder, Appl. Phys. A, Mater. Sci. Process. 63, 557 (1996).

[23] J. P. Rabe and S. Buchholz, Appl. Phys. Lett. 58, 702 (1991).

[24] P. Avouris, T. Hertel, and R. Martel, Appl. Phys. Lett. 71, 285 (1997).

[25] H. Sugimura, T. Uchida, N. Kitamura, and H. Masuhara, J. Phys. Chem. 98, 4352 (1994). 